Revue d'histoire de l'Amérique française

REVUE D'HISTOIRE DE L'AMÉRIQUE FRANÇAISE

\title{
L’enseignement de la philosophie au Petit Séminaire de Québec (1765-1880) (suite et fin)
}

\section{Marc Lebel}

Volume 19, numéro 2, septembre 1965

URI : https://id.erudit.org/iderudit/302467ar

DOI : https://doi.org/10.7202/302467ar

Aller au sommaire du numéro

Éditeur(s)

Institut d'histoire de l'Amérique française

ISSN

0035-2357 (imprimé)

1492-1383 (numérique)

Découvrir la revue

Citer cet article

Lebel, M. (1965). L'enseignement de la philosophie au Petit Séminaire de Québec (1765-1880) (suite et fin). Revue d'histoire de l'Amérique française, 19(2), 238-253. https://doi.org/10.7202/302467ar d'utilisation que vous pouvez consulter en ligne. 


\title{
L'ENSEIGNEMENT DE LA PHILOSOPHIE AU PETIT SÉMINAIRE DE QUÉBEC * \\ (1765-1880)
}

\author{
(suite et fin)
}

\section{CHAPITRE TROISIÈME}

\section{LA MONTÉE DU THOMISME (1850-1880)}

Notre histoire serait incomplète, si nous ne la menions à sa fin évidente: la promulgation par Léon XIII de l'encyclique Aeterni Patris en 1879. On voit pendant ces trente années (1850-1880) comment s'est faite au Séminaire la transition de la vieille philosophie des collèges au thomisme. L'enseignement passe peu à peu, au cours de cette période, de l'influence française à l'influence romaine. Il nous semble que les jeux se jouent durant la décennie 1860-1870.

En Europe, le retour à la scolastique débute vers le milieu $\mathrm{du}$ siècle. Jusqu'en 1850, traditionalistes et continuateurs du rationalisme chrétien s'accordent "pour renoncer à trouver dans saint Thomas la philosophie chrétienne requise par les exigences intellectuelles du temps". ${ }^{1}$ Il suffira de quelques années, après 1851, pour que le traditionalisme revienne sur sa condamnation de la scolastique et que s'opère l'étonnante mutation du traditionalisme français en néo-thomisme. L'artisan, l'initiateur en France de cette restauration fut le Père Ventura de Raulica. ${ }^{2}$ En 1851, paraît le premier manuel de philosophie scolastique du

* Voir notre Revue, XVIII : 405-424, 582-593; XIX: 106-125.

${ }^{1}$ Louis Foucher, La philosophie catholique en France au XIXe siècle avant la renaissance thomiste et dans son rapport avec elle 1800-1880 (Paris, 1955), 238.

2 Ibid., 239. 
siècle: la Philosophie de Goudin. ${ }^{3}$ Grâce aux Sanseverino, Liberatore, Talamo et Gonzalez, l'Italie et l'Espagne ne préparent aussi à la renaissance du thomisme. Vers 1860, le thomisme devient dominant dans le Collège romain. En Allemagne, le jésuite Kleutgen publie un grand traité de philosophie scolastique, bientôt traduit en français. ${ }^{4}$

A la vérité, un ensemble de circonstances, où se croisent la religion, la politique et la curiosité historique pour les choses du moyen âge, favorisent dans l'Église le retour à la scolastique. Les systèmes modernes n'ont engendré qu'un vain éclectisme, quand ils ne se sont pas tournés contre l'Église elle-même. Les entreprises récentes de catholiques bien intentionnés n'ont que trop échoué. Il existe une doctrine en tous points conforme au dogme et capable de faire l'unité des esprits: c'est le thomisme. Venant après le Syllabus, le concile du Vatican et la définition de l'infaillibilité pontificale, l'encyclique Aeterni Patris achève intellectuellement "le grand œuvre d'unité et de centralisation romaine repris au XIX ${ }^{\mathrm{e}}$ siècle". ${ }^{5}$

Que devient l'enseignement de la philosophie au Séminaire? Le programme d'études de 1854 assigne à la classe de mathématiques ou classe junior l'étude de la philosophie et des mathématiques. Il précise à propos de notre discipline:

\section{"Philosophie intellectuelle et morale. Bouvier". ${ }^{6}$}

Par philosophie intellectuelle et morale, on entend communément l'étude "des substances spirituelles, de la nature morale

3 En réalité, il s'agit de la réédition d'un vieux manuel publié pour la première fois en 1671: Philosophia juxta inconcussa tutissimaque divi Thomae dogmata. L'ouvrage est mieux connu sous le nom de son auteur, le dominicain Antoine Goudin.

4 Sur la renaissance de la scolastique, voir Joseph Louis Perrier, The Revival of Scholastic Philosophy in the Nineteenth Century (New York, 1909) et, surtout, Edgar Hocedez, Histoire de la théologie au XIX $X^{e}$ siècle II, III (Bruxelles, 1952, 1947).

5 Foucher, op. cit., 252. On trouvera le texte latin et une traduction française de l'encyclique Aeterni Patris dans Actes de Léon XIII: Encycliques, Motu Proprio, Brefs, Allocutions, Actes des Dicastères, etc.... I, (Paris, s.d.), 42-75. $55,19$.

6 Catalogue des officiers et des élèves du Séminaire de Québec, 1854- 
de l'homme, de ses rapports avec son créateur, avec ses semblables et avec lui-même. ${ }^{7}$ Notons surtout que la Philosophie de Bouvier sert de manuel de classe au Séminaire. ${ }^{8}$ A la même époque, plusieurs maisons d'éducation, dont les Séminaires de Sainte-Thérèse ${ }^{\theta}$ et de Saint-Hyacinthe ${ }^{10}$, utilisent ce manuel. Assurément, le destin de la Philosophie de Bouvier est curieux: bien qu'elle fût connue au Séminaire dès sa publication en 1824, elle ne devint manuel de classe que longtemps plus tard, certainement après $1850 .{ }^{11}$

L'histoire de la philosophie fait son entrée dans les programmes en 1857.12 Nous savons bien peu de choses sur son compte. L'Abeille, en tout cas, la dit très utile en dépit des erreurs et des absurdités révoltantes qui l'encombrent: à y bien regarder, l'histoire de la philosophie est source de vérité:

Le philosophe qui veut pénétrer jusqu'au fond des choses ... se plaît à observer la marche de l'intelligence dans le cours des siècles. Il s'efforce par de laborieuses recherches de découvrir le germe qui a donné naissance à un système ... son génie supérieur y glane des pensées profondes, ces observations judicieuses dont il saura plus tard faire son profit en les alliant avec les siennes, ou en faisant sortir les jets de lumière qui éclaireront les siècles futurs. ${ }^{13}$

${ }^{7}$ L'Abeille, 13 novembre 1851.

8 Son titre véritable est: Institutiones philosophicae ad usum seminariorum et collegiorum. La Philosophie de Bouvier, d'abord publiée en 3, puis en 1 volume, connut 12 éditions (1824-1858).

9 Emile Dubois, Le Petit Séminaire de Sainte-Thérèse (1825-1925), (Montréal, 1925), 120, 369.

10 Lucien Beauregard, La part de M. Isaac-Stanislas Désaulniers à l'introduction du thomisme au Canada francais vers l'époque de la renaissance religieuse de 1840 à 1855, in Rapport de la Soc. can. Hist. Egl. (1941$42), 80,83$.

11 On se servit probablement de la dixième édition, publiée en 1853. L'abbé Mignon écrit à propos de la Philosophie de Bouvier: "Pour le fond des choses (elle) ne sort pas de la doctrine courante des écoles cartésiennes". Voir son article: La Philosophie cartésienne dans le clergé de France aux débuts du XIX'e siècle, in Revue du clergé français, XX $(1899), 145$.

12 Officiers, professeurs et élèves du Séminaire de Québec pour l'année 1857-58, in Annuaire de l'Université Laval pour l'année académique 1858$59,55$.

13 L'Abeille, 22 juin 1859. L'article s'intitule: Utilité de l'histoire de la philosophie et analyse des principaux systèmes orientaux. A cette époque, 
Les programmes de 1862 et des années postérieures ne mentionnent pas l'histoire de la philosophie. Il n'est pas certain pour autant qu'on cesse de l'enseigner; nous verrons plus loin que l'Académie Saint-Denys et la Société Laval entendent assez régulièrement de 1860 à 1880 des travaux qui relèvent de l'histoire de la philosophie.

Assez tôt, le manuel de Bouvier est abandonné. En 1859, après une trêve de vingt-cinq ans, on retourne à la pratique des cahiers manuscrits. Un rédacteur de L'Abeille soupèse le nouveau régime:

Nous ne dirons pas tous les avantages d'un tel mode d'enseignement [le cours magistral] pour des élèves avancés en classe. Quand il n'y aurait que l'obligation de rédiger des notes prises au cours, ce serait déjà beaucoup; cela permet en effet de recueillir une foule de remarques qui ne se trouvent pas dans les livres, et puis on a la satisfaction d'être auteur. - Mais noblesse oblige dit un vieux dicton français et ce n'est pas le moindre de nos soucis; car cela donne diablement de l'ouvrage. ${ }^{14}$

Un règlement du 10 avril 1860 permet aux philosophes de première année de faire leurs classes à l'Université en suivant les cours élémentaires de la Faculté des Arts. ${ }^{15}$ A la fin du premier semestre de 1861, ils subissent un examen, "conformément aux règles de l'Université".16 L'enseignement est magis-

on connaît au Séminaire l'œuvre de Cousin: l'abbé Taschereau y fait allusion: ASQ M 339 (1845), 10; L'Abeille du 30 décembre 1859 contient un long compte rendu des Fragments et Souvenirs de Cousin. L'auteur écrit: "M. V. Cousin occupe un rang distingué, sinon parmi les hommes vraiment dignes du nom de philosophes, du moins parmi les bons écrivains français de notre siècle".

14 Ibid., 3 novembre 1859. Souligné dans le texte. Les physiciens suivent des cours oraux à l'Université depuis 1858. Rappelons que bien avant son affiliation à la Faculté des Arts en 1863, le Séminaire oblige ses élèves à se présenter aux épreuves du baccalauréat; à partir de 1858: Honorius Provost, Historique de la Faculté des Arts de l'Université Laval 1852-1952 (Québec, 1952), 7, 14.

15 Annuaire de l'Université-Laval pour l'année académique 1860-61, 19; Voir aussi Officiers, professeurs et élèves du Séminaire de Québec pour l'année 1860-61, in Annuaire de l'Université-Laval pour l'année académique 1861-62, 35 .

16 L'Abeille, 7 février 1861. 
tral, les étudiants ont d'énormes cahiers de philosophie, véritables corpus qui atteignent jusqu'à 500 pages. A partir de 1862 , les programmes ajoutent aux matières scientifiques étudiées en seconde année de philosophie (année du baccalauréat) une revision du cours de philosophie intellectuelle et morale suivi l'année précédente ${ }^{17}$.

Mais, pas plus que le manuel de Bouvier, le cours magistral ne dure guère. A son retour de Rome, en 1866, l'abbé LouisHonoré Paquet introduit le manuel de Salvatore Tongiorgi, professeur au Collège romain et apôtre de la restauration de la scolastique. ${ }^{18}$ C'est la fin de la vieille philosophie des collèges, tour à tour représentée au Séminaire dans les manuels de Toul et de Hauchecorne, de Demers et de Bouvier: finis l'éclectisme et les longues citations françaises. A l'avenir, l'influence romaine ne cessera de croître; ce sont voyages et envois d'étudiants à Rome, achats d'ouvrages scolastiques, sabbatines, etc.

De 1866 à 1879, le programme de philosophie ne varie pas. Durant les deux dernières années de leur cours, les élèves entendent 260 leçons de philosophie contre 200 de mathématiques et 410 de sciences. ${ }^{19}$ Ils ont en main le manuel de Tongiorgi; le professeur l'explique, le commente et parfois le complète par des notes dictées: chaque glose est alors accompagnée dans la marge du cahier d'un numéro qui renvoie à l'ouvrage de Tongiorgi. "Vide auctorem ad numerum designatum", écrit en $\mathbf{1 8 6 6}$ au seuil de son cahier un élève consciencieux (ou affligé d'une mémoire peu fidèle).$^{20} \mathrm{Il}$ arrive cependant que le cours se réduise à bien moins: un écolier spirituel raconte qu'en l'absence de l'abbé Paquet, souvent malade, son assistant

17 Officiers, professeurs et élèves du Séminaire de Québec durant l'année 1862-63, in Annuaire de l'Université-Laval pour l'année académique 1863-64, 59.

18 David Gosselin, Les étapes d'une classe au Petit Séminaire de Québec 1859-1868 (Québec, 1908), $178 \mathrm{~s}$. La première édition, en 3 volumes, des Institutiones philosophicae de Tongiorgi parut à Rome en 1861-1862. 19 Annuaire de l'Université Laval pour l'année académique 1871-72, 119; Annuaire de l'Université Laval pour l'année académique 1879-80, 93. 20 ASQ M 212 (1866). 
traduisait le texte, se dispensait de tout commentaire, et chaque élève philosophait en son particulier, du mieux qu'il pouvait. ${ }^{21}$

Depuis 1859, un exercice nouveau, la sabbatine, complète l'enseignement. Tous les samedis, les élèves soutiennent des thèses de philosophie en présence de leur professeur. ${ }^{22}$ Désormais les programmes d'études feront une place à des "exercices d'argumentations". ${ }^{23}$ En plus de ces sabbatines, la classe junior s'exerce à des dissertations philosophiques. Afin peut-être de bien préparer ses philosophes au baccalauréat, le Séminaire fait placer vers 1863 une bibliothèque spécialisée dans la classe de philosophie. ${ }^{24}$

Épreuve redoutable que le second examen du baccalauréat. A l'origine, trois séances de quatre heures chacune sont consacrées à la philosophie. ${ }^{25}$ Bientôt, les écoliers n'écrivent plus qu'une seule dissertation sur un sujet de logique, de métaphysique ou de morale, "selon que le sort décide". ${ }^{26}$ A partir de 1873, plutôt que de faire disserter les candidats, on leur pose plusieurs courtes questions. ${ }^{27}$

Regardons de plus haut notre sujet. D'aucuns attribuent maintenant à l'étude de la philosophie une noblesse particulière: sorte d'inflation dont les premiers signes apparaissent vers 1850 , au moment où semblent se durcir l'opposition au XVIII ${ }^{e}$ siècle et la haine du "philosophisme". Tandis qu'on attaque l'Encyclo-

21. David Gosselin, op. cit., 179.

22 L'Abeille, 6 décembre 1859 . On signale le sujet de deux récentes sabbatines: "Quelle est la meilleure définition du jugement? et, Quelle est la perception externe ?"

23 Officiers, professeurs et élèves du Séminaire de Québec durant l'année 1862-63, in Annuaire de l'Université-Laval pour l'année académique 1863-64, 59.

24 Plumitif du Séminaire, 25 mars 1863; Officiers, professeurs et élèves du Séminaire de Québec durant l'année 1862-63, in Annuaire de l'Université-Laval pour l'année académique 1863-64, 59.

25 Annuaire de l'Université-Laval pour l'année académique 1856-57, 25. Les étudiants doivent écrire "une dissertation ayant rapport à la logique; $(\ldots)$ une sur un point de métaphysique générale ou particulière; (...) une sur un point de morale".

26 Annuaire de l'Université-Laval pour l'année académique 1859-60, 23.

${ }^{27}$ Annuaire de l'Université-Laval pour l'année académique 1873-74, 77. Voir aussi l'annuaire des années postérieures. 
pédie, "vaste cloaque renfermant toutes les immondices du dixhuitième siècle" ${ }^{28}$, qu'on dénonce "le rateau niveleur de la philosophie" ${ }^{29}$, qu'on s'indigne des résultats du rationalisme ${ }^{30}$, on prête à la philosophie - à la philosophie chrétienne - un rôle considérable. De solides études philosophiques protègent de l'irréligion; la philosophie est le complément indispensable d'un bon cours. Comment combattre les impiétés de "notre prétendu siècle des lumières" ${ }^{31}$, sinon par beaucoup de philosophie ? Un rédacteur de L'Abeille reprend à son compte en 1852 l'aphorisme de Bacon:

Qu'y a-t-il... de plus important pour un jeune homme qui a fait un cours d'études que de connaître les vérités fondamentales de la religion qu'il professe, que d'être en état de répondre aux questions astucieuses du philosophisme? ... l'irréligion fait des progrès rapides et menace de toutes parts ... Un grand nombre de jeunes gens [se jettent] tête baissée dans un abîme d'erreurs... Peu de philosophie éloigne de la religion... Beaucoup de philosophie ramène à la religion. ${ }^{32}$

Il faut croire que l'abbé Hospice Verreau exprime une opinion courante lorsqu'il prononce en 1873 une conférence sur "l'importance des études philosophiques". ${ }^{33}$ Un décret du sixième concile provincial tenu à Québec en 1878 recommande aux collèges de donner à leurs élèves une solide formation philosophique. ${ }^{34}$ De nouveau, en 1881, l'Abeille fait l'éloge de la philosophie; les lauriers philosophiques ont un "cachet spécial de grandeur et de noblesse"; la philosophie est plus sérieuse que la littérature

${ }^{28}$ L'Abeille, 18 décembre 1851.

29 Ibid., 20 décembre 1849. Tiré d'un Discours sur l'histoire moderne écrit par l'abbé Joseph S. Raymond du Collège de Saint-Hyacinthe. L'Abeille des 22 novembre et 13 décembre 1849 reproduit les deux premières parties de ce Discours.

30 Ibid., 12 décembre 1850. Voir aussi l'Abeille du 22 février 1849.

31 Loc. cit.

32 Ibid., 18 novembre 1852. Souligné dans le texte.

33 Voir le compte rendu que donne le Journal de l'Instruction publique, XVII (juillet-août 1873), 122.

34 Acta et decreta sexti concilii provinciæ Quebecensis in Quebecensi civitate anno domini MDCCCLXXVIII celebrati a sancta sede revisa et recognita, (Quebeci, 1882), $42 \mathrm{~s}$. 
"où l'imagination joue souvent le rôle principal"; la philosophie est à la fois "la base et le couronnement des études: nous ne saurions y attacher trop d'importance". ${ }^{35}$

On peut penser que l'encyclique Aeterni Patris trouve au Séminaire un terrain tout préparé à reconnaître la nécessité de fortes études philosophiques. Les esprits sont-ils aussi prêts à se conformer à la méthode et à l'enseignement de saint Thomas ?

A vrai dire, on ne connaît saint Thomas que depuis peu. Sans doute le catalogue de la bibliothèque préparé en 1782 renferme-t-il une vieille édition de la Philosophie de Goudin ${ }^{36}$, mais les professeurs de l'époque ne paraissent pas s'en être servis. M. Demers ne parle nulle part de saint Thomas ou des grands scolastiques. C'est en 1848 que nous rencontrons la première allusion à saint Thomas. Cette année-là, l'abbé Taschereau fait à l'Institut canadien un "discours sur l'origine de la société et du pouvoir politique d'après saint Thomas d'Aquin". ${ }^{37}$ Six ans plus tard, il se rend à Rome où il conquiert un doctorat en théologie. Par la suite, le Séminaire et l'Université envoient assez régulièrement à Rome de jeunes professeurs pour étudier la philosophie et la théologie. Ce sont les Bégin et les Paquet (1863), les Chandonnet (1865), les Gouin, les Lemieux et les Paquet (1880), les Mathieu (1882), les Lortie (1891). A eux revient le mérite d'avoir assuré la renaissance de la scolastique, puis du thomisme.

Avec l'introduction du manuel de Tongiorgi en 1866 commence un enseignement nettement scolastique. L'auteur, il est vrai, défend une sorte d'atomisme contraire à la théorie de la matière et de la forme. Qu'importe, le prestige et l'autorité de saint Thomas grandissent à vue d'œil. Les écoliers parlent de lui comme du philosophe qu'ils suivent. ${ }^{38}$ A la demande du

${ }^{35}$ L'Abeille, 15 juin 1881.

36 Infra., appendice $\mathbf{B}$.

37 'Abeille, 22 décembre 1848 . Un peu plus tôt, vers 1840 , au Collège de Saint-Hyacinthe, l'abbé Désaulniers s'était initié au thomisme, grâce à un exemplaire de la Philosophie de Goudin: Lucien Beauregard, op. cit., 81 .

38 ASQ, Académie St-Denys. Règlement. Constitution. Procès-verbaux, II: 25 (séance du 9 février 1869); voir aussi, plus loin, II: 66 (séance du 13 février 1870). 
collège théologique de Rome, l'Université célèbre avec éclat en 1874 le sixième centenaire de la mort de saint Thomas; le Père Bourgeois et l'abbé Paquet font alors l'éloge du thomisme. ${ }^{39}$ Quelque temps plus tard, le Séminaire se procure les grands ouvrages de Jouin ${ }^{40}$ et de Kleutgen. ${ }^{41}$ On comprend donc que le pas qui sépare la scolastique du thomisme est franchi incontinent dès la promulgation de l'encyclique, le 4 août 1879. Un mois s'est à peine écoulé que l'annaliste du Séminaire inscrit cette décision du Conseil:

Résolu de conformer l'enseignement philosophique à la dernière encyclique de Léon XIII.42

Le préfet des études commande aussitôt des manuels de philosophie "d'après saint Thomas" ${ }^{43}$ et, en 1880, l'ouvrage thomiste du cardinal Zigliara, professeur à la Minerve, succède à celui de Tongiorgi. ${ }^{44}$ Bientôt, on retouche l'organisation du cours de philosophie. En 1882, la philosophie est confiée non plus à un, mais à deux professeurs: le premier voit la logique et la métaphysique, le second, la morale. ${ }^{45}$ A partir de 1884 , la classe de physique consacre chaque jour une heure à la philosophie. ${ }^{46}$ L'étude de celle-ci se répartit désormais sur deux ans:

39 Sixième Centenaire de saint Thomas d'Aquin à Saint-Hyacinthe et à Québec, (Québec, 1874). Les discours du Père Bourgeois et de l'abbé Paquet sont reproduits en entier: $23-40 ; 43-53$.

40 Plumitif du Séminaire, 14 janvier 1878. Le Père Louis Jouin, professeur au St. John's College (aujourd'hui l'Université de Fordham), publia les premiers ouvrages scolastiques en Amérique: Elementa philosophicx moralis (1865); Compendium logicx et metaphysicæ (1869). (1868).

41 Ibid., 31 mars 1879. Il s'agit sans doute de la Philosophie scolastique

42 Ibid., 9 septembre 1879. Dans une lettre circulaire du 10 novembre 1879 , l'archevêque de Québec exhorte le clergé et "les hautes maisons d'éducation" à suivre l'enseignement de saint Thomas: Mandements des évêques de Québec, VI: 183.

43 Plumitif du Séminaire, 19 janvier 1880.

44 Annuaire de l'Université Laval pour l'année académique 1881-82, 67. Lors du congrès de l'enseignement secondaire tenu en juin 1880, le comité de philosophie suggère l'emploi par tous les collèges d'un manuel uniforme: celui de Zigliara, Université 99, No $47 \mathrm{a}$.

45 Voir, en dépit du titre, ASQ, Supérieurs, Directeurs, Officiers et professeurs du Séminaire de Québec de 1663 à 1860.

46 Plumitif du Séminaire, 22 juin 1884. 


\section{Première année: philosophie (logique et métaphy- sique) ... \\ seconde année: philosophie (théologie naturelle et morale. ${ }^{47}$}

Ainsi débute et s'enracine au Séminaire une fidélité nouvelle: l'enseignement de la philosophie thomiste. Il aura suffi de quelques années pour que soit abandonnée l'ancienne philosophie des collèges et pour que les esprits se rallient à la scolastique, puis au thomisme. A l'occasion de la fête de saint Thomas en 1881, l'abbé Mathieu, professeur de philosophie au Séminaire, exalte le thomisme:

... la philosophie de saint Thomas est la seule qui par la solidité de ses principes, par l'abondance de ses lumières, par l'exactitude de ses définitions, par la force de ses raisonnements, a décidé toutes les questions, résolu tous les problèmes, éclairci toutes les erreurs, établi, rangé et mis dans leur plus grand jour, toutes les vérités; par conséquent, elle est et sera toujours la seule vraie philosophie.48

Que de différences entre 1850 et 1880 ! Ni les compendium, ni les manuels de M. Demers ou de Bouvier ne parlent de la cosmologie. Elle apparaît vers 1860 dans les cours magistraux que suivent à l'Université les élèves du Séminaire. ${ }^{49}$ L'ouvrage de Tongiorgi et celui de Zigliara consacrent par la suite l'étude de la cosmologie. A d'autres égards aussi, la transition paraît très nette. Nous le voyons bien à la considération que se méritent tour à tour le français et le latin. En 1851, L'Abeille loue la langue française et "l'admirable clarté, pour laquelle les philosophes l'ont adoptée". ${ }^{50}$ Deux ans plus tard, un correspondant renchérit:

Il n'y a peut-être pas de langue aussi nette, aussi claire, que celle dont se sont servis Descartes, Mallebranche (sic) et de Bonald. On chercherait vaine-

47 Annuaire de l'Université Laval pour l'année académique 1885-86, 38.

${ }^{48}$ L'Annuaire de l'Université Laval pour l'année académique 1881-82 reproduit en entier la conférence de l'abbé Mathieu: 53-68. (Notre citation se trouve à la p. 55). L'Abeille du 10 mars 1881 donne de larges extraits.

49 Voir par exemple ASQ M 183 (1861) : 374-395.

50 L'Abeille, 18 décembre 1851. 
ment chez les autres nations cette clarté dans les expressions, cette lucidité dans l'exposition qu'on trouve dans les écrits de ces philosophes. [...] par sa clarté et son style si logique, la langue française mérite d'être appelée la langue de la philosophie. ${ }^{51}$

En 1880, cette opinion n'a plus cours. Non seulement les sabbatines exercent-elles l'intelligence, affirme L'Abeille, mais elles familiarisent avec la langue latine, "la langue philosophique par excellence". ${ }^{52}$

Dans la réalité, au niveau de l'enseignement lui-même, quelle est la langue de la philosophie ? Observons d'abord que les manuels mis entre les mains des élèves sont rédigés en latin et que, pendant la brève période des cahiers manuscrits (185966), "le cours de philosophie se donne en latin". ${ }^{53}$ Contrairement aux Institutiones de l'abbé Demers et de Bouvier, les cahiers manuscrits et, plus tard, les manuels de Tongiorgi et de Zigliara ne renferment pas de citations françaises. En revanche, le latin n'est pas si connu des élèves que les professeurs puissent l'utiliser à l'exclusion du français. A la suite d'un examen oral de métaphysique, lors duquel il fut interrogé tantôt en français, tantôt en latin, un écolier avoue candidement:

Que de fois, ô belle langue de Cicéron et de Virgile, tu fus maltraitée ou plutôt estropiée dans ce jour néfaste ! 54

En vérité, pendant l'emploi du manuel de Tongiorgi (186680 ), il arrive souvent que les notes supplémentaires écrites par les élèves le soient en français. ${ }^{55}$ Tout comme l'assistant de l'abbé Paquet ${ }^{56}$, plusieurs professeurs traduisent en classe le texte latin: simple souci pédagogique, car c'est le latin qui, de

51 Ibid., 10 mai 1853.

52 Ibid., 25 mars 1880. C'est nous qui soulignons.

53 Officiers, professeurs et élèves du Séminaire de Québec durant l'année 1862-63, in Annuaire de l'Université-Laval pour l'année académique 1863-64, 59.

54 L'Abeille, 27 avril 1859. Souligné dans le texte.

55 Voir par exemple ASQ M 212 (1866) et M 992 (1876). Les questions du baccalauréat sont également rédigées en français.

56 Supra, 74. 
plus en plus à mesure que nous approchons de la restauration du thomisme, apparaît comme la langue par excellence de la philosophie. A la rentrée de 1878, l'abbé Mathieu prévient ses élèves :

Les cours se donnent en latin. La langue française ne se prête pas du tout au langage philosophique et théologique..$^{57}$

Plusieurs raisons "militent pour l'usage de la langue latine dans l'étude de la philosophie". Le latin favorise la précision, tandis que "les facilités, les faiblesses de la langue vulgaire, ses familiarités et ses mollesses nécessaires sont bien plus favorables au bavardage et à la divagation, ces fléaux de l'enseignement". Puisque le latin est la langue de la scolastique, nous devons l'employer; au reste, l'enseignement philosophique étant "une préparation directe et immédiate à l'enseignement théologique" qui se fait et se fera toujours en latin, c'est aussi dans cette langue que doit s'enseigner la philosophie. ${ }^{58}$

Transition très nette. Avant que ne soit introduit la scolastique en 1866, L'Abeille ne ménage pas ses louanges à l'adresse de certains philosophes modernes. Un rédacteur célèbre, en 1853, Descartes, Pascal, Fénelon, Bossuet, Malebranche et de Bonald:

Ces grands hommes ont porté les sciences morales et métaphysiques au plus haut point qu'il soit permis à l'homme d'atteindre. ${ }^{59}$

L'année suivante, nous lisons une vive critique d'Aristote (le moyen âge trouve son compte aussi) à qui on reproche d'avoir attribué aux bêtes une âme sensitive:

Ce sentiment acquit au moyen âge une telle autorité que quiconque eût osé critiquer Aristote eût commis un attentat... c'était un moyen bien simple de résoudre la plupart des difficultés... l'opinion d'Aristote ainsi que Bayle le prouve très bien ne saurait se soutenir sans entraîner dans des conséquences embarrassantes... ce système qui

57 ASQ M 333 (1878), feuillet. (Cahier de l'abbé Mathieu).

58 ASQ M 336 (1878-1882), feuillet. (Cahier de l'abbé Mathieu).

${ }^{59}$ L'Abeille, 10 mai 1853. 
s'était acquis une puissance si colossale dans le moyen âge s'écroule aussitôt qu'on en recherche les fondements, et n'a plus de force que dans le nom de son fondateur et dans le nombre de ceux qui l'ont aveuglément accepté. C'est un système arbitraire qui ne repose sur aucune vérité première et que la logique condamne. ${ }^{60}$

En 1860, un élève de philosophie flétrit à son tour le moyen âge, "siècles de ténèbres, d'ignorance où tout l'enseignement consistait dans un peu de théologie, quelques commentaires sur les décrétales des Papes, un galimatias qu'on décrétait du nom de philosophie, et enfin, quelques notions de grammaire...".61

En 1880, il n'est plus personne qui défende de telles opinions. Le plus grand respect entoure le moyen âge et la scolastique; seule la philosophie de saint Thomas apparaît capable d'arrêter le débordement de faux principes et de doctrines malsaines qui caractérisent le siècle. ${ }^{62}$ Suivant l'abbé Mathieu, la méthode scolastique constitue un excellent moyen pour calmer le désordre des intelligences et mettre un frein à l'incohérence des opinions. ${ }^{63}$ Les sabbatines "répandent comme un parfum du moyen âge". ${ }^{64}$ Certes, nos jeunes philosophes semblent lire encore après 1866 , de Bonald, ${ }^{65}$ mais leur bibliothèque contient maintenant un bon nombre d'ouvrages scolastiques. ${ }^{66}$

$\mathrm{Au}$ vrai, n'oublions pas les écoliers. Leur témoignage est fort intéressant, bien qu'on n'en puisse tirer de conclusion. Si nous écoutons J.-Edmond Roy, qui fit sa philosophie en 1875, les élèves de la cour des grands causent de "toute autre chose

60 Ibid., 30 juin 1854.

61 Ibid., 4 mai 1860.

62 Ibid., 28 avril 1881. Voir l'ensemble des Lettres de Rome que publie l'Abeille en 1880-81; on retiendra aussi le long compte rendu de la fête de saint Thomas au Séminaire de Chicoutimi, ibid., 24 mars, 31 mars 1881. 63 Conférence de l'abbé Mathieu sur saint Thomas d'Aquin, in Annuaire de l'Université Laval pour l'année académique 1881-82, 64 $\mathrm{s}$.

64 L'Abeille, 25 mars 1880.

65 ASQ, Académie St-Denys. Règlement. Constitution. Procès-verbaux, II: 25 (séance du 9 février 1869).

66 Bibliothèque - Petit Séminaire de Québec - Règlement et catalogue 1881 (Québec, 1881). 
que de Tongiorgi". ${ }^{67}$ L'abbé Mathieu, pour sa part, soutient que "les écoliers ont coutume de regarder l'année de philosophie comme une année de repos". ${ }^{68}$

Plus précieuse pour notre propos est la contribution des élèves de philosophie à l'Académie Saint-Denys et à la Société Laval. Toutes deux entendent des travaux philosophiques. Lors des séances solennelles de la première, les académiciens-philosophes lisent des essais qui le plus souvent roulent sur l'âme et sur la Providence ${ }^{69}$; on disserte aussi sur le scepticisme et sur la liberté ${ }^{70}$; Lamennais et le traditionalisme sont fréquemment refutés. ${ }^{71}$ Bien peu de philosophes présentent des travaux, notent cependant les secrétaires successifs de l'Académie. On ne voit pas assez de philosophes "descendre dans la lice, et concourir dans les tournois de l'intelligence" que prépare l'Académie. ${ }^{72}$ Ils répugneraient à dévoiler "les ténèbres sublimes de la logique et de la métaphysique". ${ }^{33}$

Lorsqu'ils se rendent à l'Académie, les philosophes sont accueillis avec chaleur. En 1869, le secrétaire se réjouit d'un afflux soudain de travaux philosophiques:

Depuis longtemps, l'Académie déplorait l'absence des élèves de philosophie dans les concours littéraires auxquels elle invite toutes les classes. Un préjugé semblait y régner, on eut dit, qu'habitués à la concision désespérante et au peu d'ornements que l'on remarque dans Aristote et dans saint Thomas d'Aquin, nos confrères semblaient croire que la raison rejetait les fleurs, et que la Muse n'était pas sœur de la philosophie; mais la lecture de Platon, de Fénelon et de Bonald nous paraît avoir fait tomber ce préjugé; car voici que les disciples de sainte Catherine descendent des hauteurs escarpées

67 J.-Edmond Roy, Souvenirs d'une classe au Séminaire de Québec (1867-187\%), (Lévis, 1905), 287.

68 ASQ M 333 (1878), feuillet.

69 ASQ, Académie St-Denys. Règlement. Constitution. Procès-verbaux, II : $53,77,120$.

70 Loc. cit.

71 Ibid., II : 66 ; III : $10,72$.

72 Ibid., II : 12.

73 Ibid., III : 51. Aussi, ibid., II : 146. 
de la science philosophique et viennent donner la main aux champions de l'éloquence et de la poésie. ${ }^{\mathbf{7 4}}$

La Société Laval entend, elle aussi, des travaux philosophiques. On y parle du scepticisme, du miracle, de l'existence de Dieu, des absurdités philosophiques du dix-neuvième siècle, de Descartes et de "son but philosophique", de la libre pensée, de la philosophie allemande, d'Épicure, du nihilisme, de la société. ${ }^{75}$

Comme il sied, la qualité de philosophe procure des privilèges, le plus estimé étant celui de préparer la fête de sainte Catherine. Bals, comédies, bâtons de tire, pommes, discours, chansons, chœurs: voilà ce que ramène annuellement la sainteCatherine:

Quand on est philosophe il est bien permis de se dérider au moins une fois par année... La philosophie n'est jamais si populaire que le jour où elle remplace les syllogismes, les dilemmes et les sorites par des bonbons et des dragées. ${ }^{76}$

\section{CONCLUSION}

Vers la fin du XIX $X^{e}$ siècle, l'enseignement de la philosophie repart à neuf. Tandis que disparaît la vieille "philosophie des collèges", la scolastique prend son essor. Bientôt, par la volonté de Rome, une doctrine parfaitement constituée rallie les esprits: le thomisme. Depuis lors, c'est de saint Thomas d'Aquin que se réclame l'enseignement; c'est lui que suivent les manuels mis entre les mains des élèves, qu'il s'agisse de celui du cardinal Zigliara ou de ceux des abbés Lortie et Grenier.

L'adhésion au thomisme est donc un fait relativement récent. Le Séminaire dispense aujourd'hui un enseignement qui fut introduit pendant les dernières décennies du XIX $\mathrm{XIècle.}^{e}$ Jusqu'en 1866, les élèves n'entendent point parler de saint Thomas; à partir de 1880, ils utilisent un manuel nettement thomiste.

74 Ibid., II : 25.

75 ASQ, Procès-verbaux de la Société Laval, II : 137, 213; III : 154, 213, 221; IV: 127 s. Voir aussi les comptes rendus de séances que donne l'Abeille: en particulier ceux des 19 février, 4 mars $1880 ; 31$ mars, 13 avril 1881.

${ }^{76} L$ 'A beille, 29 novembre 1877. 
Nous pourrions écrire la même chose des collèges classiques affiliés à l'Université Laval. Notre enseignement du thomisme est né à la fin du siècle dernier. L'encyclique Aeterni Patris a déterminé un mouvement général vers la philosophie de saint Thomas. Grâce en grande partie à ces apôtres que furent $\mathrm{Mgr}$ Louis-Adolphe Paquet, les abbés Stanislas Lortie et Arthur Robert, tous trois du Séminaire de Québec, le thomisme est devenu la philosophie de l'enseignement secondaire.

Par le thomisme s'est réalisée l'unité de l'enseignement philosophique. Fini l'éclectisme des Institutiones de M. Demers ou de l'abbé Bouvier. Des générations d'élèves ont connu la philosophie par le thomisme, y ont puisé une même vision du monde. Nous pouvons, sans préjuger le fond, parler d'une sorte de consensio: œuvre d'unité dont l'histoire très intéressante reste à écrire.

Il est certain qu'il faut examiner l'accueil qui fut fait à l'encyclique Aeterni Patris en 1880 et les interprétations qu'on a données de celle-ci depuis. Qu'attendait-on au juste de l'encyclique ? Devons-nous voir dans la diffusion rapide du thomisme un fait de discipline ecclésiastique ? Quels furent les résultats de l'unité d'enseignement? L'introduction du thomisme intéresse autant l'histoire religieuse que l'histoire de l'enseignement. C'est un événement considérable qui déborde largement l'enceinte des collèges; il vaudrait la peine de l'étudier.

En un temps où s'accumulent les projets de réformes, l'on sent le défaut d'études sur le passé de notre enseignement secondaire. Qui reprendra la synthèse tentée par l'abbé Groulx, il y a déjà plus de trente ans ? Quand publiera-t-on un livre réfléchi sur l'histoire de l'enseignement au Séminaire de Québec ? $\mathrm{Ne}$ soyons pas trop pressés cependant de servir le présent. L'histoire de l'enseignement doit survivre aux enquêtes et aux commissions et devenir une plante vigoureuse et libre.

MARC LEBEL,

Licencié ès lettres.

(Voir appendices, sous la rubrique Bibliographie). 